\title{
Very-long-chain polyunsaturated fatty acids in the retina: analysis and clinical relevance in physiological and pathological conditions
}

\author{
Olivier BERDEAUX ${ }^{1}$ \\ Niyazi $\mathrm{ACAR}^{2}$ \\ ${ }^{1}$ ChemoSens Platform, \\ Centre des Sciences du Goût et de \\ I'Alimentation, \\ UMR6265 CNRS, \\ UMR1324 INRA, \\ Université de Bourgogne, \\ Dijon, \\ F-21065 Dijon cedex \\ $<$ Olivier.Berdeaux@dijon.inra.fr $>$ \\ 2 Eye and Nutrition Research Group, \\ Centre des Sciences du Goût et de \\ I'Alimentation, \\ UMR6265 CNRS, \\ UMR1324 INRA, \\ Université de Bourgogne, \\ Dijon, \\ F-21065 Dijon cedex
}

\begin{abstract}
The retina is one of the vertebrate tissues with the highest content in polyunsaturated fatty acids (PUFA). A large proportion of retinal glycerophospholipids, especially those found in photoreceptor membranes, are dipolyunsaturated molecular species. Among them, dipolyunsaturated phosphatidylcholine (PC) molecular species are known to contain very-long-chain polyunsaturated fatty acids (VLC-PUFA) from the $n-3$ and $n-6$ series having 24-36 carbon atoms and four to six double bonds. Recent interest in the role played by VLC-PUFA arose from the findings that a protein called Elongation of very-long-chain fatty acids 4 (ELOVL4) is involved in their biosynthesis and that mutations in the ELOVL4 gene are associated with Stargardt-like macular dystrophy (STGD3), a dominantly inherited juvenile macular degeneration leading to vision loss. In this context, PC molecular species containing VLC-PUFA in retina must be precisely characterized to improve our understanding of the pathogenesis of STGD3. For 25 years, several current approaches using gas chromatography or gas chromatography-mass spectrometry have been used for the characterisation and quantification of VLC-PUFA. These methods allowed characterizing with precision the structures (carbon chain length and double bond positions) of the different VLC-PUFA in bovine retina. Thus, retinal C28-C36 fatty acids are polyunsaturated and belong to the $n-3$ and $n-6$ families. The $n-6$ family of C28-C36 fatty acids has four or five double bonds, while the $n-3$ family of VLC-PUFA contains five or six double bonds. Moreover, some studies allowed determining the position of VLC-PUFA in the VLC-PC species. But most of these conventional approaches are timeconsuming, requiring successive extraction, chromatographic steps and often a derivatization step before. Recently, a normal LC-ESI-MS/MS method was proposed for the structural characterization and the quantification of VLC-PC species in retinas from bovines and human donors. It directly analyses phospholipids as intact molecules and preserves the information based on the relative position of acyl radicals on the glycerol backbone. Since the results from molecular and biochemical studies led to the conclusion that VLC-PUFA are involved in the pathogenesis of STGD3, this specific and accurate method may be useful to more precisely investigate the molecular mechanisms leading to photoreceptor dysfunction and death in STD3.
\end{abstract}

Key words: very-long-chain polyunsaturated fatty acids, phosphatidylcholine, retina, Stargardt-like macular dystrophy type 3: STGD3, gas chromatography-mass spectrometry, liquid chromatography-mass spectrometry

branes, consist of dipolyunsaturated molecular species. Studies have reported that dipolyunsaturated phosphatidylcholine (PC) molecular species present in both rod- and cone-dominant retinas contain C22:6n-3 as one of the acyl chains, the other one being very-longchain (C24-C36) polyunsaturated fatty acids (four, five or six double bonds, VLCPUFA) (Aveldano, 1987; Aveldano and

To cite this article: Berdeaux O, Acar N. Very-long-chain polyunsaturated fatty acids in the retina: analysis and clinical relevance in physiological and pathological conditions. OCL 2011; 18(5): 284-290. doi : 10.1684/ocl.2011.0406 


$$
\begin{aligned}
& \text { VLC-PUFA, 34:6n-3 } \\
& \text { DHA, 22:6n-3 }
\end{aligned}
$$

Figure 1. Structure of a phosphatidylcholine molecule containing C34:4n-3 and docosahexaenoic acid (C22:6n-3) in the sn-1 and sn-2 positions respectively.

Sprecher, 1987; Poulos, 1995). Several studies have reported that only PC contain C28-C36 VLC-PUFA (Aveldano, 1988; Suh et al., 1994; Suh and Clandinin, 2005; McMahon, Jackson et al., 2007). Docosahexaenoic acid (DHA, 22:6n-3) tends to be located at the $s n-2$ position of the glycerol backbone while VLC-PUFA tend to locate predominantly at the $s n-1$ position (figure 1) (Aveldano, 1988). The majority of these PC species containing VLC-PUFA (named in this presentation VLC-PC) are localized in photoreceptor outer segments where the phototransduction reactions take place (Rotstein and Aveldano, 1988). In bovine photoreceptor outer segments, VLC-PC species are significant components of lipid membranes since the C28-C36 VLCPUFA represent $10 \mathrm{~mol} \%$ of total fatty acids in PCs (Aveldano, 1987).

Retinal C28-C36 VLC-PUFA belong to the $\mathrm{n}-3$ and $\mathrm{n}-6$ families (Aveldano and Sprecher, 1987) and they are synthesized in situ (Rotstein and Aveldano, 1988; Suh et al., 1994). The predominant $n-3$ polyunsaturated C28-C36 fatty acids in rat retina have been shown to be synthesized from EPA, and not from DHA (Suh and Clandinin, 2005). A protein called Elongation of very-longchain fatty acids 4 (ELOVL4) is involved in their biosynthesis (Rotstein and Aveldano, 1988; Agbaga et al., 2008). Based on sequence homology with ELOVL1, 2, 3, and 5 proteins, which are implicated in the elongation of saturated, monounsaturated, or polyunsaturated fatty acids (PUFA) from 18 to 26 carbons, the ELOVL4 protein was predicted to have similar functions (Tvrdik et al., 2000; Zhang et al., 2001; Leonard et al., 2004; Meyer et al., 2004; Westerberg et al., 2004; Agbaga et al., 2010). The ELOVL4 protein was shown to synthesize VLC-PUFA with carbon chain length of C28 and certainly longer (C30-C36) in cultured cells expressing transgenic ELOVL4 gene (Agbaga, Brush et al. 2008) and in mouse retina (McMahon et al., 2007).

\section{VLC-PUFA and Stargardt- like macular dystrophy type 3}

Recent interest in the functions played by VLC-PUFA arose from findings showing that alterations in the ELOVL4 gene in patients suffering from Stargardt-like macular dystrophy type 3 (STGD3), which is a dominantly inherited juvenile macular degeneration. These patients are affected by a severe vision loss due to large areas of macular atrophy in their retina (Zhang et al., 2001). These findings are completed by other data obtained in monkeys and showing that the ELOVL4 mRNA is exclusively expressed in cone photoreceptors that are known to be concentrated in the macular region of the retina (Umeda et al., 2003). Since at least three different mutations in the ELOVL4 gene have been identified in STGD3 patients (Edwards et al., 2001; Zhang et al., 2001; Grayson and Molday, 2005; Agbaga et al., 2010), this suggests that retinal health is highly dependent on the presence of C28-C36 PUFA, in addition to that of DHA (SanGiovanni and Chew, 2005).

In parallel to these ophthalmologic, genetic, and biochemical studies, both ELOVL4 knockout and knockin mice have been generated in order to understand the molecular mechanisms by which mutations in the ELOVL4 gene contribute to this eye disease. Within knockout models, homozygous mice had normal prenatal retinal development but were unusable after birth since they died after several hours of living due to defective skin permeability. This is consistent with a depletion of epidermal ceramides that are known to be rich in VLC saturated and monounsaturated fatty acids (Cameron et al., 2007; Li et al., 2007). Mice that were heterozygous for ELOVL4 gene displayed reduced ELOVL4 mRNA levels in their tissues but developed very normally with regular retinal function- ality and minimal morphological alterations in their retinal photoreceptors (Raz-Prag et al., 2006; Li et al., 2007). Other groups have generated and studied knockin mice expressing mutant forms of ELOVL4 gene, carrying for example the human pathogenic 5-bp deletion in the ELOVL4 gene (Karan et al., 2005; McMahon et al., 2007; Vasireddy et al., 2009). The animals were characterized by a retinal phenotype resembling that of human STGD3, including an early selective deficiency in retinal C28C36 acyl PCs, followed by a reduced retinal functionality evaluated by electroretinography, an increased accumulation undigested phagosomes and lipofuscin containing toxic N-retinylidene-N-retinylethanolamine (A2E) and a degeneration of photoreceptor cells in the central retina.

According to these findings, the proposed pathogenesis of human STGD3 is based on the alteration of photoreceptor outer segments (corresponding to the distal part of retinal photoreceptor cells) composition in VLC-PUFA affecting phototransduction processes and leading to the accumulation of toxic $A 2 E$ and further to photoreceptor death (McMahon and Kedzierski, 2010). However, and even if the relationship between VLC-PUFA and STGD3 is now well established, the exact functions of VLC-PUFA in retinal health remain unclear.

\section{Analysis of VLC-PUFA in retina}

In this context, dipolyunsaturated PC molecular species containing VLC-PUFA in retina must be precisely characterized to improve our understanding of the pathogenesis of STGD3. Several current approaches were used for the characterization and quantification of VLC-PUFA or VLC-PC in biological samples. Gas chromatography-mass spectrometry equipped with electronic ionization (GC-ElMS) and Liquid-chromatography - tandem mass spectrometry equipped with an electrospray ionization interface (LCESI-MS/MS) have been shown to be the most reliable analytical approaches to characterize and quantify VLC-PUFA or VLC-PC in biological samples and in particular in the retina.

The VLC-PUFAs were initially characterized in PC from bovine retina by Aveldaño and co-workers (Aveldano, 1987; 


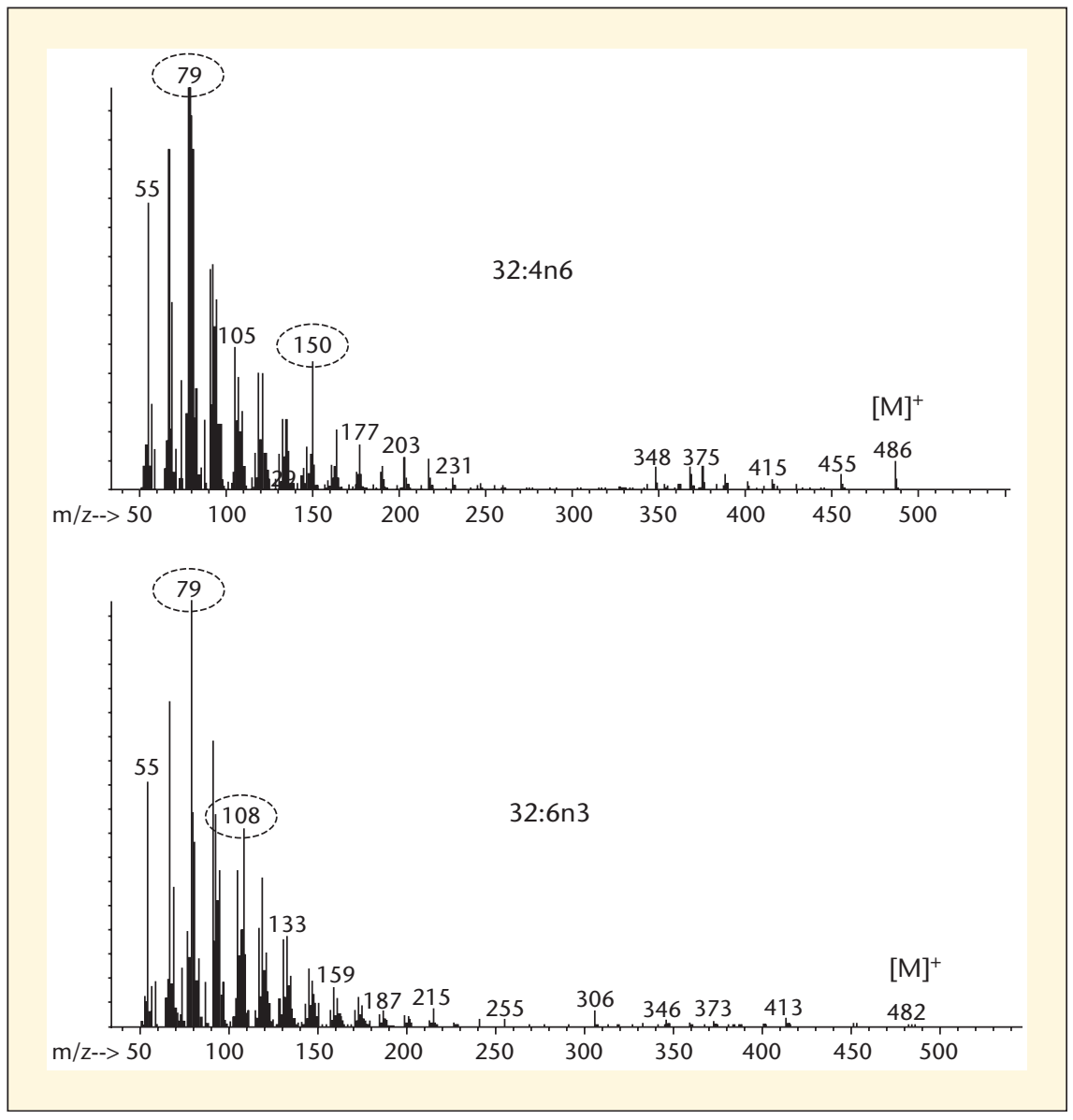

Figure 2. Electron impact mass spectra and total ion of 34:4 (n-6) and 32:6 (n-3) methyl esters (Suh et al., 1994; Agbaga et al., 2008). Reproduced by kind permission of Proceeding of the National Academy of Sciences and redrawn from the original publication.

Aveldano and Sprecher, 1987). In their study, PC were purified by TLC, converted into acetyldiglycerides and resolved into groups of molecular species

(fraction of similar unsaturation) by means of argentation thin layer chromatography $\left(\mathrm{AgNO}_{3}-\mathrm{TLC}\right)$. VLC-PUFA were purified and separated by successive

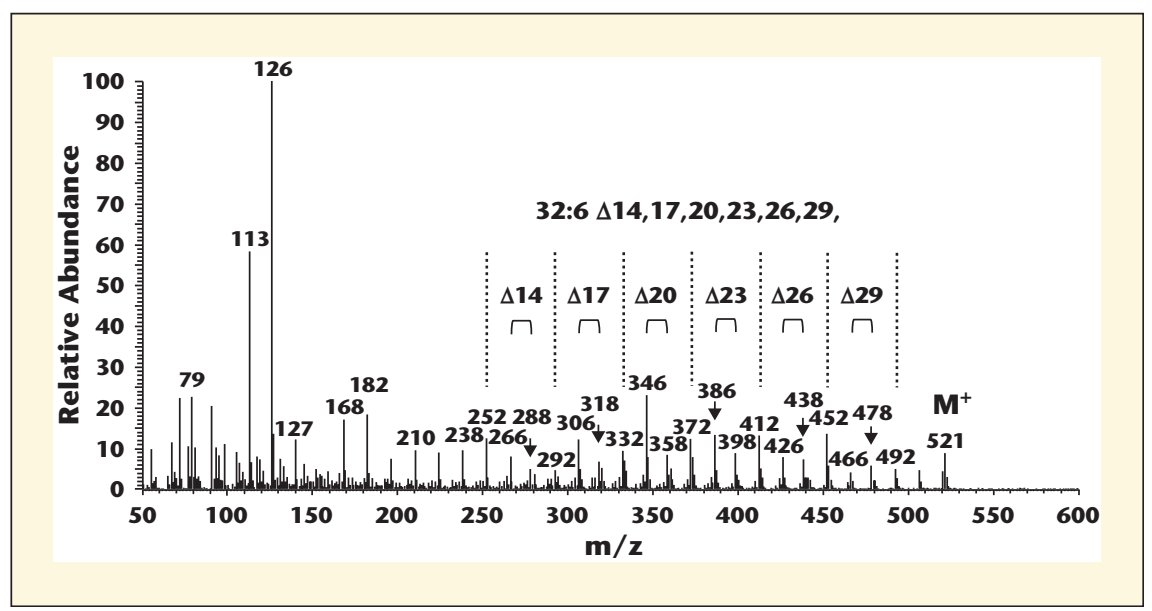

Figure 3. Mass spectra of 4,4-dimethyloxazoline (DMOX) derivatives of 32:6n-3 (Berdeaux et al., 2010). Reproduced by kind permission of Journal of Chromatography A and redrawn from the original publication. chromatographic steps ( $\mathrm{TLC}, \mathrm{AgNO}_{3}-$ TLC and HPLC), and converted into fatty acid methyl esters (FAME) derivatives. FAMEs were then characterized with precision using a combination of oxidative ozonolysis and GC-EI-MS analyses. Using oxidative ozonolysis for the localization of the double bond, it was shown that very long chain tetraenes belonged to the $n-6$ series, hexaenes to the $n-3$ series, and major pentaenes to the $n-3$ series of fatty acids but very long chain $n$ 6 pentaenes also occurred. Molecular ions were obtained by GC-EI-SM of FAME (figure 2) which conclusively identified the major VLC-PUFA in bovine retina. Suh and co-workers developed a similar GCEI-MS methodology to quantify VLCPUFA. In their study, they aimed to determine the effect of altering diet fat composition on the long-chain-PUFA and VLC-PUFA content of individual phospholipids of the photoreceptor outer segments (ROS) in normal and diabetic animals (Suh et al., 1994). VLC-PUFA were isolated from the total lipid extract from ROS by successive chromatographic steps and converted to FAME derivatives. $\mathrm{AgNO}_{3}-\mathrm{TLC}$ was used to resolve FAMEs on the basis of degree of unsaturation and FAMEs of each band were injected in GC-El-MS analyses. Fatty acid identifying was confirmed by molecular ion $\left[\mathrm{M}^{+}\right.$ identification. In addition, GC-El-MS provided definitive identification of the series of PUFA containing diagnostic ion at $m / z 79$ as the base pic (figure 2). Moreover, mass spectra of PUFA containing a significant fragment at $m / z 108$ are from the $n-3$ series whereas those containing a significant ion at $\mathrm{m} / \mathrm{z} 150$ are from the $\mathrm{n}-6$ series. More recently, Agbaga and co-worker used GC-El-MS to study the role of ELOVL4 protein in the biosynthesis of VLC-PUFA (Agbaga et al., 2008). In this study, VLC-PUFA were first isolated from total lipids extract of bovine retina, converted to FAME derivatives and identified by GC-EI-MS as described above. In a second step, and by using mass spectra and relative retention time data, they were able to identify these fatty acids in samples containing low concentrations by the use of single ion monitoring (SIM) mass spectrometry. By monitoring the $m / z$ ratios 79,108 and 150 , they have identified and measured the VLC-PUFA from ELOVL4-expressings cells for their study.

However, for structural identification of PUFA and in particular of VLC-PUFA, 


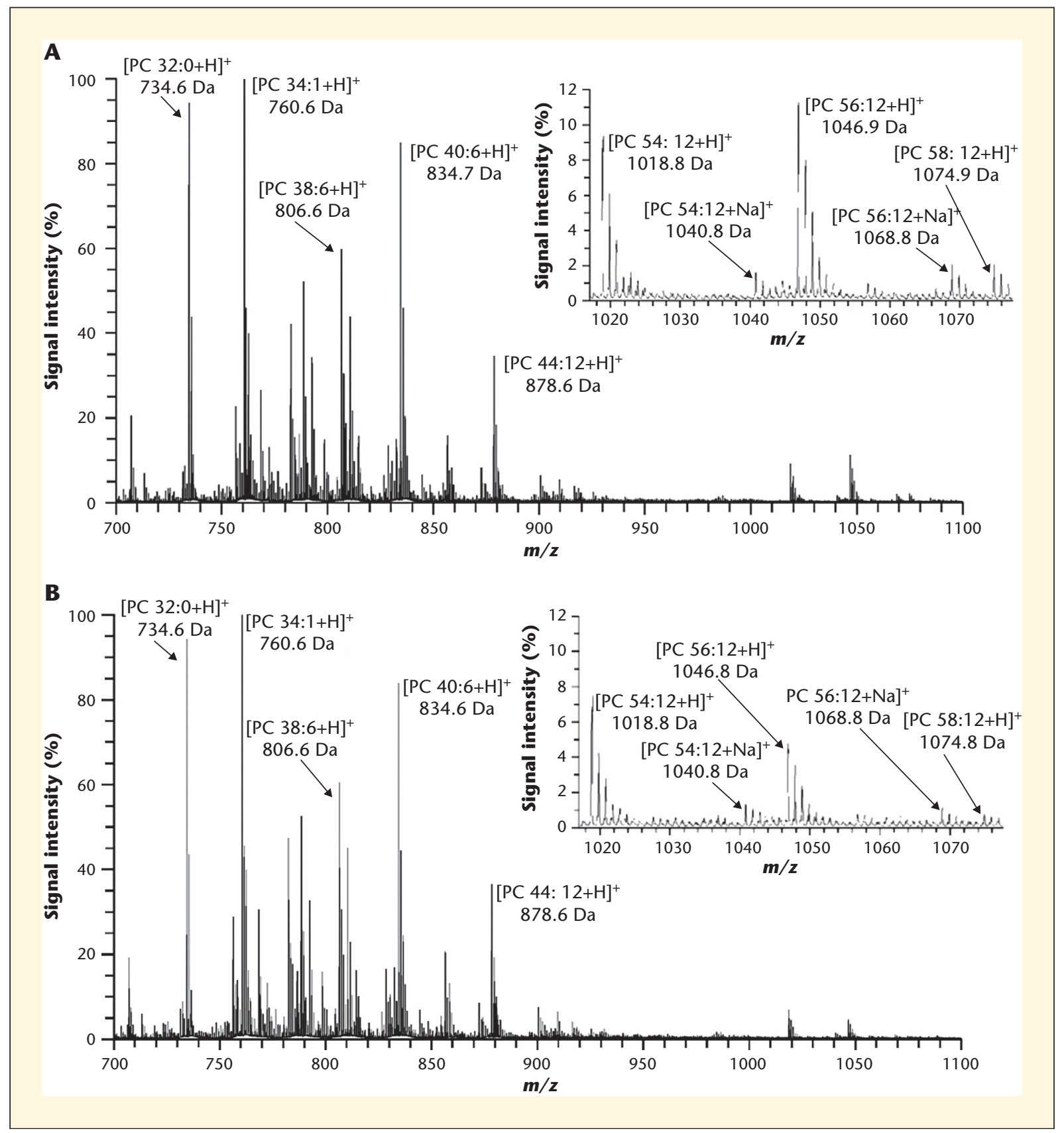

Figure 4. MS analysis of retinal lipids from (A) a 1-month-old wild-type and (B) STGD3-heterozygous mouse (McMahon et al., 2007). Reproduced by kind permission of FEBS Letters and redrawn from the original publication.

mass spectra of FAMEs not contain ions indicating the position of double bonds on the aliphatic chain. In the most useful approach to structure determination, the carboxyl group is derivatized with a reagent containing a nitrogen atom. When the molecule is ionized in the mass spectrometer, the nitrogen atom but not the alkyl chain carries the charge, and double bond ionization and migration is minimized. 4,4-Dimethyloxazoline (DMOX) derivatives give excellent mass spectra of fatty acids with electron-impact ionization that frequently permit unequivocal identification (Zhang et al., 1988; Fay and Richli, 1991; Berdeaux and Wolff, 1996). Recently, we have used DMOX derivatives for structural identification of VLCPUFA with precise localization of the double bonds along the carbon chain (Berdeaux et al., 2010). The VLC-PC fractions from bovine and human retinas were isolated by HPLC and hydrolyzed.
VLC-PUFA were then converted into DMOX derivatives and analyzed using GC-ESI-MS. For illustration, the mass spectra of the DMOX derivative of C32:6 (n-3) are presented in figure 3 . All VLC-PUFA from human retina were characterized with precision. In samples from bovine retinas, as in those from human retinas, C30-C34 VLC-PUFA of the $n-6$ family had only four double bonds, while C28-C36 VLC-PUFA of the $\mathrm{n}-3$ family had five or six double bonds 


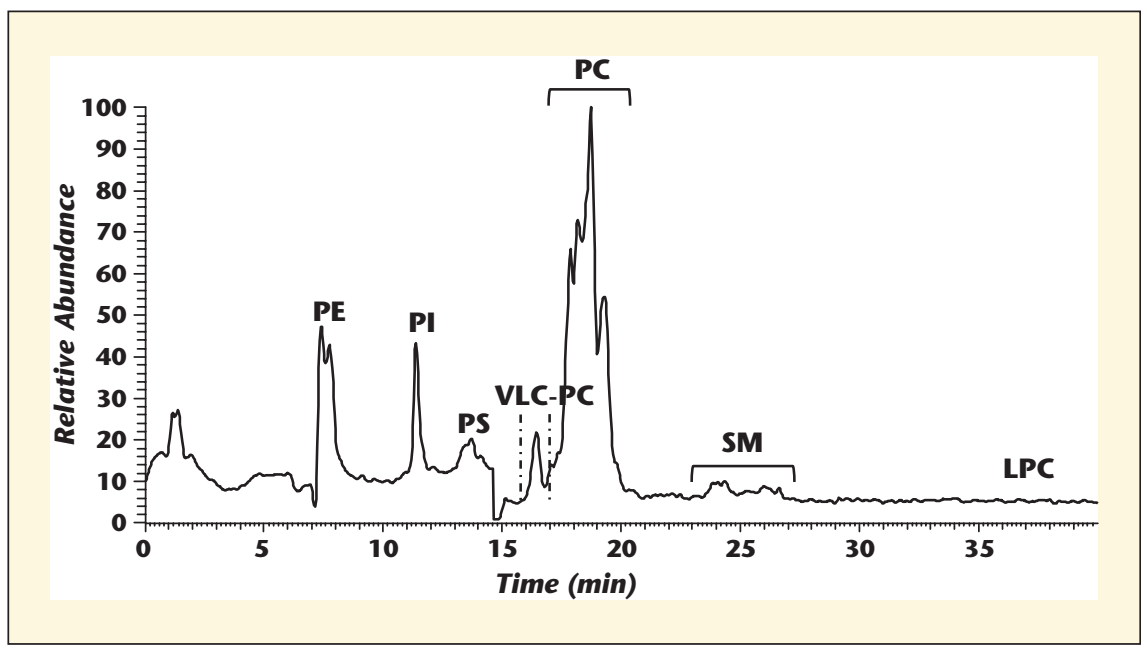

Figure 5. LC-ESI-MS normal-phase chromatogram of the lipid extract from human retina. (Berdeaux et al., 2010). Reproduced by kind permission of Journal of Chromatography $A$ and redrawn from the original publication.

according to previous studies (Aveldano, 1987; Suh et al., 1994). As expected, C32 and C34 VLC-PUFA with four, five and six double bonds seemed to represent the prominent VLC-PUFA in bovine as well as in human retinas (Berdeaux et al., 2010).

But most of these conventional approaches are time-consuming, requiring successive extraction, chromatographic steps (HPLC, TLC) and often a derivatization step before gas chromatography (GC) or gas chromatography-mass spectrometry (GC-MS) analyses.

Electrospray ionization-mass spectrometry (ESI-MS) has been described as a soft ionization technology (Kerwin et al., 1994). It is the most sophisticated

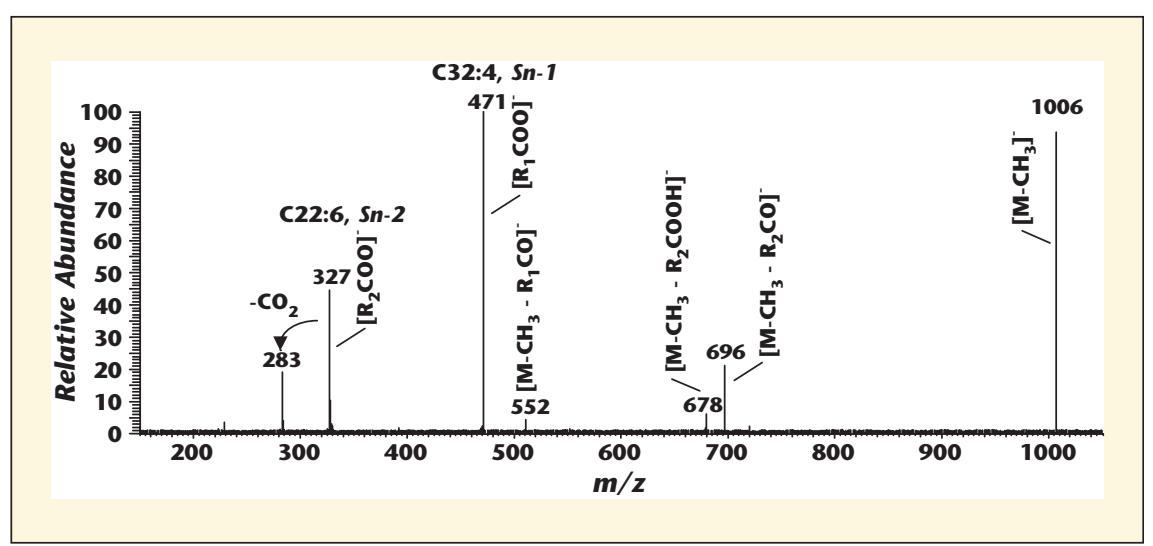

Figure 6. Product-ion spectra of negative ions of the $\left[\mathrm{M}-\mathrm{CH}_{3}\right]^{-}$of $P \mathrm{C} 32.4 / 22: 6$ at $\mathrm{m} / \mathrm{z} 1006$ showing two abundant ions at $\mathrm{m} / \mathrm{z} 327$ and $\mathrm{m} / \mathrm{z} 471$, corresponding to C22:6 and C32:4 carboxylate anions, and the product ions formed by neutral loss of either an FA or a ketene at $\mathrm{m} / \mathrm{z} 678\left(\left[\mathrm{M}-\mathrm{CH}_{3}-\mathrm{R}_{2} \mathrm{CH}-\mathrm{COOH}\right]^{-}\right), \mathrm{m} / \mathrm{z} 696\left(\left[\mathrm{M}-\mathrm{CH}_{3}-\mathrm{R}_{2} \mathrm{CH}=\mathrm{C}=\mathrm{O}\right]^{-}\right)$, and $\mathrm{m} / \mathrm{z} 552$ $\left(\left[\mathrm{M}-\mathrm{CH}_{3}-\mathrm{R}_{7} \mathrm{CH}=\mathrm{C}=\mathrm{O}\right]^{-}\right)$. molecular specie was detected as protonated molecular ions $[\mathrm{M}+\mathrm{H}]^{+}$. Moreover, to gain more detailed information on the PC structures, authors further analyzed retinal lipids in presence of $\mathrm{LiCl}$ salt. Liadducts of PC are more extensively fragmented than are the protonated PCs, thus yielding more pertinent structural information (Hsu et al., 1998). More than twenty mass peaks were detected. Among them, 3 VLC-PC, namely PC 32:6/22:6, PC34:6/22:6 and PC36:6/ 22:6 were clearly characterized. When the MS spectra of retinal lipids from STGD3-heterozygous mice were compared with the spectra of their wild-type littermates, most of the corresponding peaks were similar (figure 4). The only peaks that have had their heights reduced in mutant mouse sample belonged to C32-C36 VLC-PC. These MS analyses demonstrated that the STGD3 mutation causes selective deficiency of C32-C36 acyl PCs in mouse retina.

LC-ESI-MS was widely used for characterization and quantification of phospholipids in different types of tissues. The use of LC prior to the ESI-MS/MS analysis enhances the detection of the minor isobaric species in the mixture. Additionally, a suitable chromatographic separation may reduce any ESI suppression of non-isobaric and co-eluting species. Recently, we have developed a LC-ESIMS/MS method for the structural characterization and the quantification of VLC-PC molecular species in total lipid extracts from bovine and human retinas (Berdeaux et al., 2010). The total lipid extract was directly analyzed by LC-ESIMS/MS without purification or derivatization. A good baseline separation of phospholipid classes in bovine and human retinas was achieved using normal-HPLC conditions (figure 5). Moreover VLC-PC molecular species containing VLC-PUFA eluted separately just before the other PC molecular species. Hence, it was possible to analyze directly this fraction containing only VLC-PC for structure characterization in LC-MS or to collect this fraction in order to concentrate VLC-PC for further structure characterization using GC-MS.

When operated in the single stage MS mode in ESI-, each PC and VLC-PC molecular specie produced an abundant demethylated molecular ions [M $\left.\mathrm{CH}_{3}\right]^{-}$used for its structural characterization and its quantification. Then, a complete structural characterization of 


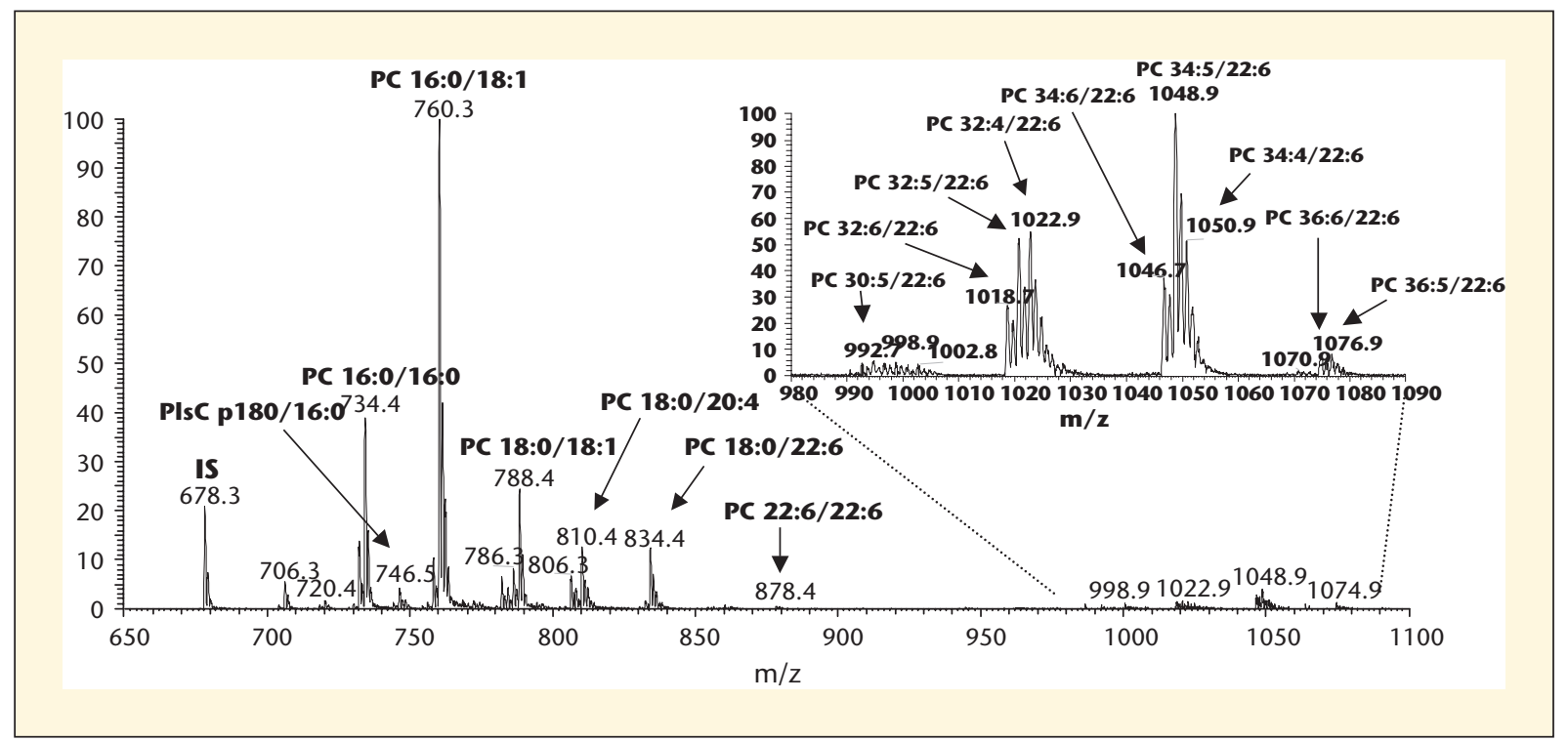

Figure 7. Positive-ion HPLC-ESI-MS mass spectra of total PC fraction collected from human neural retina by scanning for precursors at $\mathrm{m} / \mathrm{z} 184$ in the positive mode (Berdeaux et al., 2010). Reproduced by kind permission of Journal of Chromatography $A$ and redrawn from the original publication.

intact PC and VLC-PC species in retina was obtained by collision-induced dissociation (CID) in the negative mode. Indeed, the $\mathrm{MS}^{2}$ experiment of the selected demethylated molecular ions $\left[\mathrm{M}-\mathrm{CH}_{3}\right]^{-}$of PC species gave a characteristic fragmentation. Fatty acid composition and distribution could be clearly assigned based on the intensity of $s n-2 / s n-1$ fragment ions as illustrated in Figure 6 for PC 32:4/22:6 at $\mathrm{m} / \mathrm{z}$ 1006. Thus, VLC-PC species characterised in bovine and human retina were dipolyunsaturated PC species containing one VLC-PUFA (C24-C36) with three to six double bonds. Moreover, VLC-PUFA was always in the $s n-1$ position of VLC-PC whilst PUFA at the sn-2 position was exclusively C22:6. Therefore, the quantitative analysis of the different PC and VLC-PC molecular species was performed (figure 7) (Berdeaux et al., 2010). 36 PC and choline plasmalogens ( $\mathrm{Pls} \mathrm{C}$ ) species were quantified in retinas from human donors. Among them, 12 VLC-PC were detected and quantified. The main compounds represented were those having VLC-PUFA with 32 carbon atoms (C32:3, C32:4, C32:5 and C32:6) and 34 carbon atoms (C34:3, C34:4, C34:5 and C34:6). Dipolyunsaturated PCs with $36: 5$ and $36: 6$ were detected but in smaller quantities. This study showed that HPLC-ESI-MS/MS method is a valuable method for a direct and precise characterization of PC molecular species containing VLC-PUFA in retina and may be useful for a better understanding of the pathogenesis of STCD3.

\section{REFERENCES}

Agbaga MP, Brush RS, et al. Role of Stargardt3 macular dystrophy protein (ELOVL4) in the biosynthesis of very long chain fatty acids. Proc Natl Acad Sci U S A 2008; 105: 12843-8.

Agbaga MP, Mandal MN, et al. Retinal very long-chain PUFAs: new insights from studies on ELOVL4 protein. J Lipid Res 2010; 51: 1624-42.

Aveldano MI. A novel group of very long chain polyenoic fatty acids in dipolyunstaurated phosphatidylcholines from vertebrate retina. J Biol Chem 1987; 262: 1172-9.

Aveldano MI. Phospholipid species containing long and very long polyenoic fatty acids remain with rhodopsin after hexane extraction of phostoreceptor membranes. Biochemistry 1988; 27: 1229-39.

Aveldano MI, Sprecher H. Very long chain (C24 to C36) polyenoic fatty acids of the $n-3$ and $n-6$ series in dipolyunsaturated phosphatidylcholines from bovine retina. / Biol Chem 1987; 262: 1180-6.

Berdeaux $\mathrm{O}$, Juanéda $\mathrm{P}$, et al. Identification and quantification of phosphatidylcholines containing very-long-chain polyunsaturated fatty acid in bovine and human retina using liquid chromatography/tandem mass spectrometry. J Chromatogr A 2010; 1217: 7738-48.
Berdeaux O, Wolff R. Gas-liquid chromatography-mass spectrometry of the 4,4-dimethyloxazoline derivatives of D5-unsaturated polymethylene-interrupted fatty acids from conifer seed oils. J Am Oil Chem Soc 1996; 73: 1323-6.

Cameron DJ, Tong ZZ, et al. Essential role of Elovl4 in very long chain fatty acid synthesis, skin permeability barrier function, and neonatal survival. Int J Biol Sci 2007; 3: 111-9.

Edwards AO, Donoso LA, et al. A novel gene for autosomal dominant Stargardt-like macular dystrophy with homology to the SUR4 protein family. Investig Ophthalmol Vis Sci 2001; 42: 52-2663.

Fay L, Richli U. Location of double bonds in polyunsaturatde fatty acids by gas chromatography-mass spectrometry after 4,4-dimethyloxazoline derivatization. / Chromatogr 1991; 541: 89-98.

Grayson C, Molday RS. Dominant negative mechanism underlies autosomal dominant Stargardt-like macular dystrophy linked to mutations in ELOVL4. J Biol Chem 2005; 280: 32521-30.

Hsu FF, Bohrer A, et al. Formation of lithiated adducts of glycerophosphocholine lipids facilitated their identification by electrospray ionization tandem mass spectrometry. J Am Soc Mass spectrom 1998; 9: 516-26.

Karan G, Lillo C, et al. Lipofuscin accumulation, abnormal electrophysiology, and photoreceptor degeneration in mutant ELOVL4 transgenic mice: a model for macular degeneration. Proc Natl Acad Sci U S A 2005; 102: 4164-9. 
Kerwin JL, Tuininga AR, et al. Identification of molecular species of glycerophospholipids and sphingomyelin using electrospray mass spectrometry. J Lipid Res 1994; 35: 1102-14.

Leonard $\mathrm{AE}$, Pereira SL, et al. Elongation of long-chain fatty acids. Prog Lipid Res 2004; 43: $36-54$

Li W, Chen Y, et al. Elovl4 haploinsufficiency does not induce early onset retinal degeneration in mice. Vision Res 2007; 47: 714-22.

Li W, Sandhoff $\mathrm{R}$, et al. Depletion of ceramides with very long chain fatty acids causes defective skin permeability barrier function, and neonatal lethality in ELOVL4 deficient mice. Int / Biol Sci 2007; 3: 120-8.

McMahon A, Jackson SN, et al. A Stargardt disease- 3 mutation in the mouse Elovl 4 gene causes retinal deficiency of C32-C36 acyl phosphatidylcholines. FEBS Letters 2007; 581: 5459-63.

McMahon A, Kedzierski W. Polyunsaturated very-long-chain C28-C36 fatty acids and retinal physiology. $\mathrm{Br}$ J Ophthalmol 2010; 94: 1127-32.

Meyer A, Kirsch $\mathrm{H}$, et al. Novel fatty acid elongases and their use for the reconstitution of docosahexaenoic acid biosynthesis. J Lipid Res 2004; 45: 1899-909.

Poulos A. Very long chain fatty acids in higher animals-a review. Lipids 1995; 30: 1-14.

Raz-Prag D, Ayyagari R, et al. Haploinsufficiency is not the key mechanism of pathogenesis in a heterozygous Elovl4 knockout mouse model of STGD3 disease. Invest Ophthalmol Vis Sci 2006; 47: 3603-11.

Rotstein NP, Aveldano MI. Synthesis of very long chain (up to 36 carbon) tetra, penta and heaenoic fatty acids in retina. Biochem / 1988; 249: 191-200.

SanGiovanni JP, Chew EY. The role of omega3 long-chain polyunsaturated fatty acids in health and disease of the retina. Prog Retin Eye Res 2005; 24: 87-138.

Suh M, Clandinin MT. 20:5n-3 but not $22: 6 n-3$ is preferred substracte for synthesis of $n-3$ very-long-chain fatty acids (C24-C36) in retina. Curr Eye Res 2005; 30: 959-68.

Suh $M$, Wierzbicki AA, et al. Dietary fat alters membrane composition in rod outer segments in normal and diabetic rats: impact on content of very-long-chain ( $C>24$ ) polyenoic fatty acids. Biochim Biophys Acta 1994; 1214: 54-62.
Tvrdik P, Westerberg R, et al. Role of a new mammalian gene family in the biosynthesis of very long chain fatty acids and sphingolipids. I Cell Biol 2000; 149: 707-17.

Umeda S, Ayyagari R, et al. Molecular cloning of ELOVL4 gene from cynomolgus monkey (Macaca fascicularis). Experimental animals/ Japanese Association for Laboratory Animal Science 2003; 52: 129-35.

Vasireddy V, Jablonski MM, et al. Elovl4 5-bp deletion knock-in mouse model for Stargardt-like macular degeneration demonstrates accumulation of ELOVL4 and lipofuscin. Exp Eye Res 2009; 89: 905-12.

Westerberg R, Tvrdik P, et al. Role for ELOVL3 and fatty acid chain length in development of hair and skin function. J Biol Chem 2004; 279: 5621-9.

Zhang JY, Yu QT, et al. Chemical modification in mass spetrometry IV-2-alkenyl-4,4-dimethyloxazolines as derivatives for the double bond location of long-chain olefinic acids. Biomed Environ Mass Spectrom 1988; 15: 33-44.

Zhang ZQ, Wang $\mathrm{Y}$, et al. Wildtype Kras2 can inhibit lung carcinogenesis in mice. Nat Genet 2001; 29: 25-33. 